\title{
Compliance with the Surviving Sepsis Campaign guideline 1-hour bundle for septic shock in China in 2018
}

\author{
Lu Wang ${ }^{1 \#}$, Xudong $\mathrm{Ma}^{2 \#}$, Huaiwu $\mathrm{He}^{1}$, Longxiang $\mathrm{Su}^{1}$, Yanhong Guo ${ }^{2}$, Guangliang Shan ${ }^{3}$, Ye Wang ${ }^{3}$, \\ Xiang Zhou ${ }^{1}$, Dawei Liu ${ }^{1}$, Yun Long ${ }^{1}$; China National Critical Care Quality Control Center Group \\ ${ }^{1}$ Department of Critical Care Medicine, Peking Union Medical College Hospital, Peking Union Medical College and Chinese Academy of Medical \\ Sciences, Beijing, China; ${ }^{2}$ Department of Medical Administration, National Health Commission of the People's Republic of China, Beijing, China; \\ ${ }^{3}$ Department of Epidemiology and Biostatistics, Institute of Basic Medicine Sciences, Chinese Academy of Medical Sciences (CAMS) and School of \\ Basic Medicine, Peking Union Medical College, Beijing, China \\ Contributions: (I) Conception and design: X Zhou, D Liu; (II) Administrative support: Y Guo, Y Long; (III) Provision of study materials or patients: L \\ Wang, X Ma, H He, L Su; (IV) Collection and assembly of data: All authors; (V) Data analysis and interpretation: G Shan, Y Wang; (VI) Manuscript \\ writing: All authors; (VII) Final approval of manuscript: All authors. \\ "These authors contributed equally to this work. \\ Correspondence to: Xiang Zhou; Dawei Liu. Department of Critical Care Medicine, Peking Union Medical College Hospital, 1\# Shuaifuyuan, \\ Dongcheng District, Beijing 100730, China. Email: zx_pumc@126.com; daweiliu_pucmhicu@163.com.
}

Background: Effective implementation of the Surviving Sepsis Campaign (SSC) guidelines has effectively reduced sepsis mortality. The effects of hospital level and ownership on compliance with the SSC guideline 1-hour bundle $\left(\mathrm{C}_{\mathrm{ssc}-1 \mathrm{~h}}\right)$ are unclear. We designed this study to identify the differences in $\mathrm{C}_{\mathrm{ssc}-1 \mathrm{~h}}$ between secondary and tertiary hospitals, public hospitals, and private hospitals.

Methods: In this survey, 1,420 hospitals were enrolled, including 864 public tertiary hospitals, 482 public secondary hospitals, 34 private tertiary hospitals, 40 private secondary hospitals. The data were collected between January 1, 2018, and December 31, 2018. The outcomes were adherence to the SSC guidelines (2018 update). Monitoring indicators include 1-hour bundle and its sub-indicators (measure lactate level and remeasure lactate level if initial lactate is $>2 \mathrm{mmol} / \mathrm{L}$, obtain blood cultures before administering antibiotics, administer broad-spectrum antibiotics, begin rapid administration of $30 \mathrm{~mL} / \mathrm{kg}$ crystalloid for hypotension or lactate $\geq 4 \mathrm{mmol} / \mathrm{L}$, apply vasopressor if hypotension is present during or after fluid resuscitation to maintain a mean arterial pressure $\geq 65 \mathrm{mmHg}$ ). Every monitoring indicator was stratified by the median, which is defined as 1 if greater than or equal to the median, and 0 if not.

Results: $\mathrm{C}_{\mathrm{ssc}-1 \mathrm{~h}}$ in tertiary hospitals was significantly higher than in secondary hospitals $(\mathrm{P}<0.05)$. However, there were no statistical differences in $\mathrm{C}_{\text {ssc-1h }}$ in public hospitals and private hospitals.

Conclusions: $\mathrm{C}_{\mathrm{ssc}-\mathrm{h}}$ in tertiary hospitals was significantly better than that in secondary hospitals. There is an urgent need to improve $\mathrm{C}_{\mathrm{ssc}-1 \mathrm{~h}}$ in secondary hospitals. The increase in private hospitals will not reduce $\mathrm{C}_{\mathrm{ssc}-1 \mathrm{~h}}$.

Keywords: Saving Sepsis Campaign guidelines; tertiary hospitals; secondary hospitals; public hospitals; private hospitals

Submitted Jul 21, 2020. Accepted for publication Nov 20, 2020.

doi: $10.21037 /$ atm-20-5429

View this article at: http://dx.doi.org/10.21037/atm-20-5429

\section{Introduction}

Sepsis-3 is defined as the downregulation of host response after infection and the occurrence of life-threatening organ dysfunction, which is an important cause of death (1).
Septic shock is a serious condition in sepsis development, with abnormal circulation and metabolism (2). Even with active fluid resuscitation, persistent hypotension can occur and be life-threatening due to a combination of 
severe vasodilatation, low catecholamine reactivity, and myocardial inhibition in septic shock (3). Sepsis has become the most-watched disease in critical care medicine due to its high morbidity and mortality (4). The "sepsis bundle" has been central to the implementation of the Surviving Sepsis Campaign (SSC) from the first publication of its evidence-based guidelines in 2004 (5-8). The evidence in the literature has demonstrated an association between compliance with bundles and improved survival in patients with sepsis and septic shock (9-11). To improve compliance with bundles, the SSC revised their care bundle in 2018 (12). Unlike the 3 -hour and 6-hour bundle recommended in their 2016 guidelines (8), the 2018 update recommends completing the sepsis bundle within 1 hour (12).

Hospitals in China are classified into 3 levels. Primary hospitals rarely admit and treat patients with septic shock. Secondary hospitals are the main body of diagnosis and provide treatment for septic shock. Tertiary hospitals are medical technology centers in China. The main function of tertiary hospitals is to provide specialized medical services to solve critical and difficult diseases. According to ownership, hospitals in China are classified into public hospitals and private hospitals. With the adjustment of medical policies, private hospitals play an increasingly important role in diagnosing and treating septic shock. Whether there is a difference in compliance with SSC guideline 1-hour bundle $\left(\mathrm{C}_{\text {ssc-1h }}\right)$ between secondary and tertiary hospitals, public hospitals, and private hospitals has not been reported in the literature. Therefore, we conducted this survey to identify the differences in $\mathrm{C}_{\mathrm{ssc}-1 \mathrm{~h}}$ between secondary and tertiary hospitals, public hospitals, and private hospitals to improve the prognosis of patients with sepsis. We present the following article following the Materials Design Analysis Reporting (MDAR) reporting checklist (available at http:// dx.doi.org/10.21037/atm-20-5429).

\section{Methods}

\section{Study design}

In this survey, 1,420 hospitals were enrolled in the Quality Improvement of Critical Care Program, led by the China National Critical Care Quality Control Center. These comprised 864 public tertiary hospitals, 482 public secondary hospitals, 34 private tertiary hospitals, 40 private secondary hospitals. These hospitals admitted 143,616 patients with septic shock, of which public tertiary hospitals admitted 108,696 patients, public secondary hospitals admitted 31,197 patients, private tertiary hospitals admitted 1,975 patients, and private secondary hospitals admitted 1,748 patients. Data were collected between January 1 , 2018, and December 31, 2018. Informed consent was obtained from every hospital participating in the study. All information on the participating hospitals is listed in Tables 1 and 2 .

The outcomes were adherence to the SSC guidelines (2018 update). Monitoring indicators included the 1-hour bundle and its sub-indicators (measure lactate level and remeasure lactate level if initial lactate is $>2 \mathrm{mmol} / \mathrm{L}$, obtain blood cultures before administering antibiotics, administer broad-spectrum antibiotics, begin rapid administration of $30 \mathrm{~mL} / \mathrm{kg}$ crystalloid for hypotension or lactate $\geq 4 \mathrm{mmol} / \mathrm{L}$, apply vasopressor if hypotension is present during or after fluid resuscitation to maintain a mean arterial pressure $\geq 65 \mathrm{mmHg}$ ). Every monitoring indicator was stratified by the median, defined as 1 if greater than or equal to the median, and 0 if not.

Based on the data obtained from this survey, we analyzed the effects of hospital level and ownership on $\mathrm{C}_{\text {ssc-lh }}$ in septic shock in China. We then determined which medical quality indicators affected $\mathrm{C}_{\mathrm{ssc}-1 \mathrm{~h}}$ in septic shock in China.

The study was conducted following the Declaration of Helsinki (as revised in 2013). The central institutional review board approved the trial protocol at Peking Union Medical College Hospital (No. S-K1297), and individual consent for this retrospective analysis was waived.

In all participating clusters, data were obtained and entered into a web-based data entry system by a local, trained independent research coordinator who was not involved in patient care and received compensation from this trial. Range checks were used to check for inconsistent or out-of-range data and prompted the user to correct or review data entries outside the predefined range. The system also provided predefined logic checks to identify errors or illogical data entries. A data-quality meeting was held monthly to review all the hospital enrollment records and registry data. The datasets supporting the conclusions of this article are included within the article and its additional files.

\section{Statistical analysis}

Statistical analysis was performed using SPSS 16.0 software (SPSS, Chicago, IL, USA). Basic information was expressed as mean \pm standard error of mean values and 
Table 1 Basic information of tertiary and secondary hospitals

\begin{tabular}{lccc}
\hline Variables & Tertiary hospitals & Secondary hospitals & $P$ \\
\hline Public hospitals & & & $<.0001$ \\
Beds/ICU & $27.86 \pm 34.71$ & $12.14 \pm 6.99$ & $<0.0001$ \\
Patients /ICU & $1,212.54 \pm 1,717.63$ & $559.97 \pm 510.99$ & $<0.0001$ \\
Patients with septic shock/ICU & $125.81 \pm 179.61$ & $64.72 \pm 111.85$ & \\
Private hospitals & & $9.85 \pm 4.5$ & 0.0005 \\
Beds/ICU & $17.03 \pm 10.28$ & $364.7 \pm 256.82$ & 0.0009 \\
Patients/ICU & $661.18 \pm 432.08$ & $43.7 \pm 30.18$ & 0.0722 \\
Patients with septic shock/ICU & $58.09 \pm 37.65$ & & \\
\hline
\end{tabular}

Table 2 Basic information of public and private hospitals

\begin{tabular}{lccc}
\hline Variables & Public hospitals & Private hospitals & $P$ \\
\hline Tertiary hospitals & & & $<.0001$ \\
Beds/ICU & $27.86 \pm 34.71$ & $17.03 \pm 10.28$ & $<0.0001$ \\
Patients/ICU & $1,212.54 \pm 1,717.63$ & $661.18 \pm 432.08$ & $<0.0001$ \\
Patients with septic shock/ICU & $125.81 \pm 179.61$ & $58.09 \pm 37.65$ & \\
Secondary hospitals & $12.14 \pm 6.99$ & $9.85 \pm 4.5$ & 0.0048 \\
Beds/ICU & $559.97 \pm 510.99$ & $364.7 \pm 256.82$ & $<0.0001$ \\
Patients/ICU & $64.72 \pm 111.85$ & $43.7 \pm 30.18$ & 0.003 \\
Patients with septic shock/ICU & &
\end{tabular}

compared using the one-way analysis of variance test. $\mathrm{C}_{\mathrm{ssc}}$ ${ }_{1 \mathrm{~h}}$ was expressed as median and quartile $\mathrm{M}$ (P25, P75), and compared using the rank test. Stratifications of $\mathrm{C}_{\mathrm{ssc}-1 \mathrm{~h}}$ were expressed as 0 or 1 , and compared using logistic regression. $\mathrm{P}<0.05$ was considered to be statistically significant.

\section{Results}

\section{Effects of hospital level on $C_{\text {ssc-1b }}$}

In public hospitals, except for the completion of empiric broad-spectrum therapy and completion of resuscitation with $30 \mathrm{~mL} / \mathrm{kg}$ crystal liquid, most indicators of $\mathrm{C}_{\mathrm{ssc}-\mathrm{h}}$ in tertiary hospitals were significantly higher than those in secondary hospitals $(\mathrm{P}<0.05)$. The same phenomenon was not observed in private hospitals (Table 3).

\section{Effects of hospital ownership on $C_{s c-1 b}$}

In both tertiary and secondary hospitals, all indicators of
$\mathrm{C}_{\mathrm{ssc}-1 \mathrm{~h}}$ between public hospitals and private hospitals were not statistically different (Table 4).

Effects of hospital level and ownership on the stratification of $C_{s s c-1 b}$

Stratification of $\mathrm{C}_{\mathrm{ssc}-\mathrm{h}}$ in tertiary hospitals was significantly higher than that in secondary hospitals $(\mathrm{P}<0.05)$. However, there were no statistical differences in the stratification of $\mathrm{C}_{\mathrm{ssc}-1 \mathrm{~h}}$ in public hospitals and private hospitals (Table 5).

\section{Differences in medical quality index between tertiary bospitals and secondary hospitals}

The nurse-to-patient ratio in the intensive care unit (ICU) and the proportion of total ICU inpatient bed occupancy in tertiary hospitals were significantly higher than that in secondary hospitals $(\mathrm{P}<0.05)$. The same phenomenon was not observed in the doctor-to-patient ratio in the ICU 
Table 3 Effects of hospital level on $\mathrm{C}_{\mathrm{ssc}-1 \mathrm{~h}}$

\begin{tabular}{lccc}
\hline Variables & Tertiary hospitals & Secondary hospitals & P \\
\hline Public hospitals & & & \\
Hour-1 bundle & $79.29(54.48,95.52)$ & $72.55(40.85,95.24)$ & 0.0034 \\
Remeasure of lactate & $88.57(64.35,100)$ & $80.1(46.88,100)$ & $<0.0001$ \\
Blood cultures & $97.1(78.04,100)$ & $86.36(54.12,100)$ & $<0.0001$ \\
Broad-spectrum antibiotics & $100(91.73,100)$ & $100(88.37,100)$ & 0.4324 \\
30 mL/kg crystalloid & $94.31(79.45,100)$ & $92.45(75.36,100)$ & 0.1929 \\
Vasopressor & $80(57.6,100)$ & $68.3(41.67,93.75)$ & $<0.0001$ \\
Private hospitals & & & 0.4364 \\
Hour-1 bundle & $70.43(47.83,100)$ & $70(45.43,97.37)$ & 0.5383 \\
Remeasure of lactate & $72.64(45.21,100)$ & $78.71(53.13,100)$ & 0.393 \\
Blood cultures & $97.87(69.7,100)$ & $83.67(54.57,100)$ & 0.1986 \\
Broad-spectrum antibiotics & $100(90.91,100)$ & $100(76.94,100)$ & 0.8846 \\
30 mL/kg crystalloid & $90.75(70.18,100)$ & $92.37(70.5,100)$ & 0.2887 \\
Vasopressor & $75.8(54.1,100)$ & $63.33(48.11,98.11)$ & \\
\hline
\end{tabular}

Remeasure of lactate = measure lactate level and remeasure lactate if initial lactate elevated $(>2 \mathrm{mmol} / \mathrm{L})$; blood cultures = obtain blood cultures before administering antibiotics; broad-spectrum antibiotics = administer broad-spectrum antibiotics; $30 \mathrm{~mL} / \mathrm{kg} \mathrm{crystalloid} \mathrm{=}$ begin rapid administration of $30 \mathrm{~mL} / \mathrm{kg}$ crystalloid for hypotension or lactate $\geq 4 \mathrm{mmol} / \mathrm{L}$; vasopressor = apply vasopressor if hypotension during or after fluid resuscitation to maintain a mean arterial pressure $\geq 65 \mathrm{mmHg}$.

Table 4 Effects of hospital ownership on $\mathrm{C}_{\text {ssc-1h }}$

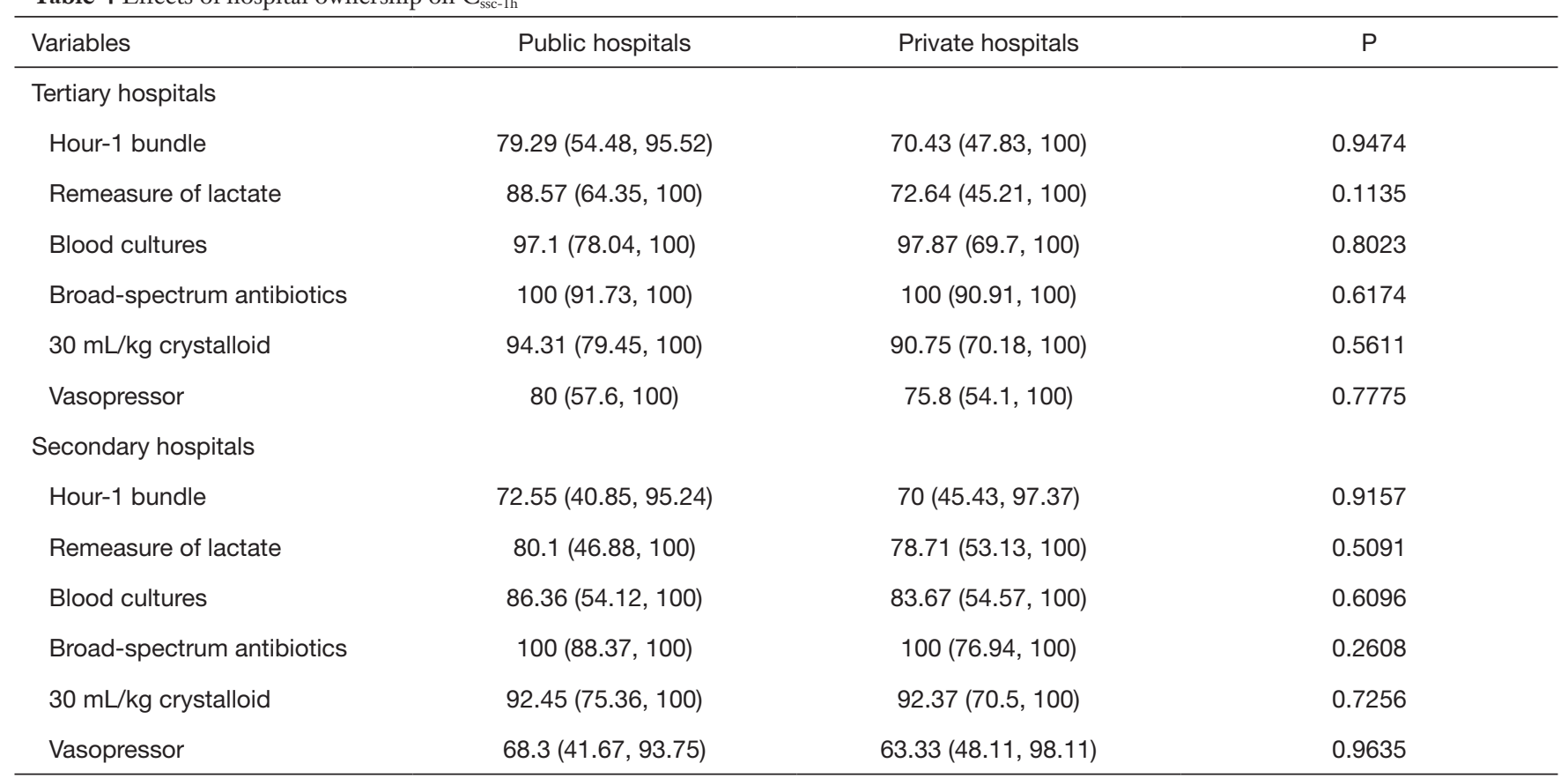

Remeasure of lactate = measure lactate level and remeasure lactate if initial lactate elevated $(>2 \mathrm{mmol} / \mathrm{L})$; blood cultures = obtain blood cultures before administering antibiotics; broad-spectrum antibiotics = administer broad-spectrum antibiotics; $30 \mathrm{~mL} / \mathrm{kg} \mathrm{crystalloid} \mathrm{=}$ begin rapid administration of $30 \mathrm{~mL} / \mathrm{kg}$ crystalloid for hypotension or lactate $\geq 4 \mathrm{mmol} / \mathrm{L}$; vasopressor = apply vasopressor if hypotension during or after fluid resuscitation to maintain a mean arterial pressure $\geq 65 \mathrm{mmHg}$. 
Table 5 Effects of hospital level and ownership on stratification of $\mathrm{C}_{\mathrm{scc}-\mathrm{hh}}$

\begin{tabular}{lcccc}
\hline \multirow{2}{*}{ Variables } & \multicolumn{2}{c}{ Tertiary vs. secondary } & \multicolumn{2}{c}{ Public vs. private } \\
\cline { 2 - 5 } & OR & $95 \% \mathrm{Cl}$ & $\mathrm{OR}$ & \multicolumn{1}{c}{$95 \% \mathrm{Cl}$} \\
\hline Hour-1 bundle & 1.442 & $1.085-1.914$ & 1.415 & $0.836-2.394$ \\
Remeasure of lactate & 1.632 & $1.206-2.209$ & 1.000 & $0.565-1.773$ \\
Blood cultures & 2.032 & $1.442-2.863$ & 0.765 & $0.391-1.497$ \\
Broad-spectrum & 2.259 & $1.385-3.684$ & & $0.404-2.473$ \\
antibiotics & & & 1.070 & $0.506-2.263$ \\
30 mL/kg crystalloid & 1.887 & $1.259-2.829$ & 1.071 & $0.619-1.852$ \\
Vasopressor & 1.715 & $1.285-2.289$ & & \\
\hline
\end{tabular}

Remeasure of lactate = measure lactate level and remeasure lactate if initial lactate elevated $(>2 \mathrm{mmol} / \mathrm{L})$; blood cultures = obtain blood cultures before administering antibiotics; broad-spectrum antibiotics = administer broad-spectrum antibiotics; $30 \mathrm{~mL} / \mathrm{kg} \mathrm{crystalloid} \mathrm{=}$ begin rapid administration of $30 \mathrm{~mL} / \mathrm{kg}$ crystalloid for hypotension or lactate $\geq 4 \mathrm{mmol} / \mathrm{L}$; vasopressor = apply vasopressor if hypotension during or after fluid resuscitation to maintain a mean arterial pressure $\geq 65 \mathrm{mmHg}$.

and Acute Physiology and Chronic Health Evaluation (APACHE II) score $\geq 15$ in all ICU patients (Table 6).

\section{Discussion}

There are at least 31.5 million sepsis patients worldwide, 19.4 million of whom have severe sepsis. More than 5 million patients died of sepsis every year (13). In the treatment of sepsis, effective implementation of SSC guidelines can reduce fatality and improve prognosis (9-11). To improve compliance with bundles, the SSC constantly updates their care bundle according to new evidence from the first publication in 2004. In 2018 the SSC launched 1-hour bundle (12). Unlike the 3-hour and 6-hour bundles that were recommended in the SSC 2016 guidelines (8), the 2018 update recommends completing the sepsis bundle within 1 hour (12). To improve $\mathrm{C}_{\mathrm{ssc}-\mathrm{hh}}$ and the prognosis of patients with sepsis, the National Health Commission of China issued quality control requirements for critical care medicine in 2015, including sepsis treatment. However, $\mathrm{C}_{\mathrm{ss}-1 \mathrm{~h}}$ in Asia is still low at present (14). Educating medical staff on sepsis being a medical emergency and that it is important to implement guidelines effectively is still a problem. There are still some doctors in China, especially non-ICU doctors, who lack understanding of SSC guidelines, and many have many problems implementing the SSC guidelines. Wang et al. found that the implementation of SSC guidelines by emergency physicians is often hindered by doctors' awareness and attitude (15). In the present study, monitoring indicators were developed according to SSC guideline 1-hour bundle to analyze differences in $\mathrm{C}_{\mathrm{ssc}-1 \mathrm{~h}}$ in different types of hospitals in China in 2018 to strengthen the supervision of sepsis treatment and implement the SSC guideline 1-hour bundle more effectively. In the present study, we found that $\mathrm{C}_{\text {ssc-1h }}$ in tertiary hospitals was significantly higher than that in secondary hospitals. However, there were no statistical differences in $\mathrm{C}_{\mathrm{ssc}-\mathrm{h}}$ in public hospitals and private hospitals.

In China, hospital qualifications are assessed according to hospital functions, facilities, technical strength, and other indicators. Hospital qualification assessment is unified across the country, regardless of hospital background or ownership. According to the hierarchical management standard of hospitals, hospitals are divided into 3 levels. Until October 2019, 2,671 tertiary hospitals accounted for $7.91 \%, 9,410$ secondary hospitals accounting for $27.88 \%$, and 11,011 primary hospitals accounting for $32.62 \%$ of all hospitals in China. Primary hospitals are primary health care institutions whose main function is to provide primary prevention directly to the population; they rarely admit and treat patients with septic shock. Therefore, primary hospitals were not included in the scope of this study. Secondary hospitals are technical centers of regional medical treatment and prevention and the main body of diagnosis and septic shock treatment. Tertiary hospitals provide transregional, provincial, and municipal medical and health services and are medical technology centers in China. The main function of tertiary hospitals is to provide specialized medical services to treat critical and difficult diseases. We conducted this survey to identify 
Table 6 Differences of medical quality indicators between tertiary and secondary hospitals

\begin{tabular}{lccc}
\hline Variables & Tertiary hospitals & Secondary hospitals & P \\
\hline Doctor to patient ratio of ICU & $0.64 \pm 0.35$ & $0.66 \pm 0.38$ & 0.27 \\
Nurse to patient ratio of ICU & $1.94 \pm 0.76$ & $1.86 \pm 0.74$ & 0.02 \\
Proportion of ICU in total inpatient bed & $0.020 \pm 0.031$ & $0.017 \pm 0.023$ & 0.02 \\
occupancy & & $0.48 \pm 0.32$ & 0.73 \\
$\begin{array}{l}\text { Proportion of APACHE II score } \geq 15 \text { in all } \\
\text { ICU patients }\end{array}$ & $0.47 \pm 0.32$ & & \\
\hline
\end{tabular}

Proportion of ICU in total inpatient bed occupancy = (days of ICU bed occupancy by patients)/(days of hospital bed occupancy by patients at the same periods); proportion of APACHE II score $\geq 15$ in all ICU patients $(\%)=($ No. of patients with APACHE II score $\geq 15$ during the first $24 \mathrm{~h}$ in ICU)/(patients admitted ICU at the same periods).

$\mathrm{C}_{\text {ssc-1h }}$ differences in the management of septic shock between secondary hospitals and tertiary hospitals. In our study, $\mathrm{C}_{\mathrm{ssc}-1 \mathrm{~h}}$ in tertiary hospitals was significantly higher than that in secondary hospitals. The reason for this could be that tertiary hospitals have greater technical and human resources. There were no statistical differences between tertiary hospitals and secondary hospitals in APACHE II score $\geq 15$ and the proportion of ICU doctors/beds in our study. However, tertiary hospital ICUs have a higher proportion of nurses/beds and total inpatient bed occupancy than secondary hospital ICUs. These data indicate that there are no differences between tertiary hospitals and secondary hospitals in the degree of critical illness of patients admitted to the ICU and that the major difference between tertiary hospitals and secondary hospitals is not the doctor configuration, but in nurse configuration and total inpatient bed occupancy in the ICU. A higher proportion of total inpatient bed occupancy in the ICU often represents a stronger voice within China's hospital.

However, secondary hospitals are the main body of septic shock treatment. Most septic shock patients are treated in secondary hospitals. Therefore, there is an urgent need to improve $\mathrm{C}_{\mathrm{ssc}-1 \mathrm{~h}}$ in secondary hospitals. According to the statistical results, there is still room for improvement in $\mathrm{C}_{\text {ssc- }-1 \mathrm{~h}}$ in secondary hospitals, and quality control should be strengthened to improve $\mathrm{C}_{\text {ss- }-1 \mathrm{~h}}$ in secondary hospitals. Increasing the proportion of nurses/beds and total inpatient bed occupancy in ICUs of secondary hospitals may be an effective way to improve $\mathrm{C}_{\text {ssc-1h }}$. In our survey on private hospitals, although most indicators of $\mathrm{C}_{\mathrm{ssc}-\mathrm{hh}}$ in tertiary hospitals were higher than those in secondary hospitals, there were no statistical differences. This could be because the number of private hospitals included in this survey was too small, and further studies are needed to determine whether there is a difference between $\mathrm{C}_{\text {ssc- }-1 \mathrm{~h}}$ in tertiary hospitals and secondary hospitals in private hospitals.

According to ownership, hospitals in China are classified into public hospitals and private hospitals. Public hospitals are non-profit and receive financial subsidies from the state, so their medical prices are strictly limited. Private hospitals are generally profit-making hospitals under the government's supervision and are responsible for their profits and losses, with independent decisions made on medical prices. Since 1980, private hospitals began to appear in China's medical industry. The large-scale development of private hospitals in China was after 2001. Due to the shortage in public hospital funds, local governments focused their medical funds on private capital. In September 2001, China opened its medical market and encouraged the development of private medical institutions. The number of private hospitals began to increase continuously since then. In 2016, the number of private hospitals exceeded that of public hospitals for the first time. Until October 2019, the number of private hospitals in China had reached 21,838 , while the number of public hospitals had been reduced to 11,914 . With an increase in the number of private hospitals, the number of patients admitted with septic shock significantly increased. As technical force is still relatively weak, the general public, and even some medical professionals, generally believe that private hospitals are the weak link in treating septic shock. To identify $\mathrm{C}_{\mathrm{ssc}-1 \mathrm{~h}}$ differences between public and private hospitals, we conducted this survey. It was surprising that there were no significant $\mathrm{C}_{\text {ssc-1h }}$ differences between private hospitals and public hospitals in our study. From this perspective, private hospitals at the same level have the same septic shock diagnosis and treatment capabilities as public hospitals, and the development of private hospitals will not reduce $\mathrm{C}_{\mathrm{ssc}-1 \mathrm{~h}}$. 
Raised blood lactate concentrations are frequently viewed as evidence of tissue hypoxia and/or oxygen debt secondary to hypoperfusion $(8,9)$. Serum lactate level is a sign of tissue hypoperfusion and an independent prognostic factor of septic shock (10). Studies have shown that lactate clearance $\geq 10 \%$ within 6 hours of resuscitation may indicate a lower case fatality rate $(11,12)$. Our previous clinical studies have shown that the ladder-oriented strategy of lactate scavenging can significantly reduce the mortality of shock patients (13-15), and we proposed to optimize the scavenging of lactate by respiratory quotient $\left[\mathrm{P}(\mathrm{v}-\mathrm{a}) \mathrm{CO}_{2} /\right.$ $\left.\mathrm{C}(\mathrm{a}-\mathrm{v}) \mathrm{O}_{2}\right](16,17)$. In public hospitals, the completion of measure lactate level and remeasure lactate level in tertiary hospitals was significantly higher than that in secondary hospitals. Both in tertiary and secondary hospitals, the completion of measure lactate level and remeasure lactate level between public hospitals and private hospitals were not statistically different. Stratification of measuring lactate level and remeasure lactate in tertiary hospitals was significantly higher than that in secondary hospitals. This phenomenon suggests that we should improve the completion of measure lactate level and remeasure lactate level in secondary hospitals.

Sterilization of cultures can occur within minutes of the first dose of an appropriate antimicrobial (16), so cultures must be obtained before antibiotic administration to optimize pathogens' identification and improve outcomes (17). Appropriate blood cultures include at least aerobic and anaerobic blood cultures. In public hospitals, the completion of obtaining blood cultures before administering antibiotics in tertiary hospitals was significantly higher than that in secondary hospitals. Both in tertiary and secondary hospitals, the completion of obtaining blood cultures before administering antibiotics between public hospitals and private hospitals was not statistically different. Stratification of completion of obtaining blood cultures before administering antibiotics in tertiary hospitals was significantly higher than that in secondary hospitals. This phenomenon suggests that we should improve the completion of obtaining blood cultures before administering antibiotics in secondary hospitals.

Empiric broad-spectrum therapy to cover all likely pathogens should be started immediately in septic shock (18). Empiric antimicrobial therapy should be narrowed once pathogen identification and sensitivities are established. The completion of empiric broadspectrum therapy between public hospitals and private hospitals, tertiary hospitals, and secondary hospitals was not statistically different. This could be because most hospitals do this well; all had a median of 100 .

Early effective fluid resuscitation is crucial for septic shock. Initial fluid resuscitation should begin immediately upon recognizing a patient with hypotension and elevated lactate and completed within 3 hours of recognition. The completion of early effective fluid resuscitation between public hospitals and private hospitals, tertiary hospitals, and secondary hospitals was not statistically different. The reason for this could be that initiating the rapid administration of $30 \mathrm{~mL} / \mathrm{kg}$ crystalloid for hypotension or lactate $\geq 4 \mathrm{mmol} / \mathrm{L}$ has been the usual practice in the early stages of resuscitation (19).

Urgent restoration of adequate perfusion pressure to the vital organs is a key part of resuscitation. This should not be delayed. If blood pressure is not restored after initial fluid resuscitation, then vasopressors should be commenced within the first hour to achieve a mean arterial pressure of $\geq 65 \mathrm{mmHg}$ (20). In public hospitals, the completion of applying vasopressors in tertiary hospitals was significantly higher than that in secondary hospitals. Both in tertiary and secondary hospitals, the completion of applying vasopressors between public hospitals and private hospitals was not statistically different. Stratification of the completion of applying vasopressors in tertiary hospitals was significantly higher than that in secondary hospitals. This suggests that we should improve the completion of applying vasopressors in secondary hospitals.

The present study has some limitations. First, only 1 year of data was included in the study, and the effects of hospital level and ownership on $\mathrm{C}_{\text {ssc-1h }}$ could not be analyzed continuously and dynamically. Second, compared with the proportion of private hospitals in terms of actual hospital composition, the number of private hospitals included in the study was relatively small, which may cause differences between survey results and real-life settings. It is necessary to conduct further studies using large samples of private hospitals.

\section{Conclusions}

$\mathrm{C}_{\text {ssc-1h }}$ in tertiary hospitals was significantly higher than that in secondary hospitals. There is an urgent need to improve $\mathrm{C}_{\text {ssc-1h }}$ in secondary hospitals. There were no statistical differences in the $\mathrm{C}_{\text {ssc-1h }}$ in public hospitals and private hospitals; therefore, the increase in private hospitals will not 
reduce $\mathrm{C}_{\mathrm{ssc}-1 \mathrm{~h}}$.

\section{Acknowledgments}

Funding: National Key R\&D Program of China (grant number 2020YFC0861000). National Natural Science Fund of China (grant number 81801901). The funders had no role in the study's design and conduct; collection, management, analysis, and interpretation of the data; preparation, review, or approval of the manuscript; and decision to submit the manuscript for publication.

\section{Footnote}

Reporting Checklist: The authors have completed the MDAR reporting checklist. Available at http://dx.doi.org/10.21037/ atm-20-5429

Data Sharing Statement: Available at http://dx.doi. org/10.21037/atm-20-5429

Peer Review File: Available at http://dx.doi.org/10.21037/ atm-20-5429

Conflicts of Interest: All authors have completed the ICMJE uniform disclosure form (available at http://dx.doi. org/10.21037/atm-20-5429). The authors have no conflicts of interest to declare.

Ethical Statement: The authors are accountable for all aspects of the work in ensuring that questions related to the accuracy or integrity of any part of the work are appropriately investigated and resolved. The study was conducted in accordance with the Declaration of Helsinki (as revised in 2013). The trial protocol was approved by the central institutional review board at Peking Union Medical College Hospital (No. S-K1297) and individual consent for this retrospective analysis was waived.

Open Access Statement: This is an Open Access article distributed in accordance with the Creative Commons Attribution-NonCommercial-NoDerivs 4.0 International License (CC BY-NC-ND 4.0), which permits the noncommercial replication and distribution of the article with the strict proviso that no changes or edits are made and the original work is properly cited (including links to both the formal publication through the relevant DOI and the license). See: https://creativecommons.org/licenses/by-nc-nd/4.0/.

\section{References}

1. Singer M, Deutschman CS, Seymour CW, et al. The Third International Consensus Definitions for Sepsis and Septic Shock (Sepsis-3). JAMA 2016;315:801-10.

2. Shankar-Hari M, Phillips GS, Levy ML, et al. Developing a New Definition and Assessing New Clinical Criteria for Septic Shock: For the Third International Consensus Definitions for Sepsis and Septic Shock (Sepsis-3). JAMA 2016;315:775-87.

3. Vieillard-Baron A, Caille V, Charron C, et al. Actual incidence of global left ventricular hypokinesia in adult septic shock. Crit Care Med 2008;36:1701-6.

4. Paoli CJ, Reynolds MA, Sinha M, et al. Epidemiology and Costs of Sepsis in the United States-An Analysis Based on Timing of Diagnosis and Severity Level. Crit Care Med 2018;46:1889-97.

5. Dellinger RP, Carlet JM, Masur H, et al. Surviving Sepsis Campaign guidelines for management of severe sepsis and septic shock. Intensive Care Med 2004;30:536-55.

6. Dellinger RP, Levy MM, Carlet JM, et al. Surviving Sepsis Campaign: international guidelines for management of severe sepsis and septic shock: 2008. Intensive Care Med 2008;34:17-60.

7. Dellinger RP, Levy MM, Rhodes A, et al. Surviving Sepsis Campaign: international guidelines for management of severe sepsis and septic shock, 2012. Intensive Care Med 2013;39:165-228.

8. Rhodes A, Evans LE, Alhazzani W, et al. Surviving Sepsis Campaign: International Guidelines for Management of Sepsis and Septic Shock: 2016. Intensive Care Med 2017;43:304-77.

9. Seymour CW, Gesten F, Prescott HC, et al. Time to Treatment and Mortality during Mandated Emergency Care for Sepsis. N Engl J Med 2017;376:2235-44.

10. Leisman DE, Doerfler ME, Ward MF, et al. Survival Benefit and Cost Savings From Compliance With a Simplified 3-Hour Sepsis Bundle in a Series of Prospective, Multisite, Observational Cohorts. Crit Care Med 2017;45:395-406.

11. Ferrer R, Martin-Loeches I, Phillips G, et al. Empiric antibiotic treatment reduces mortality in severe sepsis and septic shock from the first hour: results from a guidelinebased performance improvement program. Crit Care Med 2014;42:1749-55.

12. Levy MM, Evans LE, Rhodes A The Surviving Sepsis Campaign Bundle: 2018 update. Intensive Care Med 2018;44:925-8. 
13. Fleischmann C, Scherag A, Adhikari NK, et al. Assessment of Global Incidence and Mortality of Hospital-treated Sepsis. Current Estimates and Limitations. Am J Respir Crit Care Med 2016;193:259-72.

14. Baig MA, Shahzad H, Jamil B, et al. Sepsis management: An evidence-based approach. J Pak Med Assoc 2016;66:337-8.

15. Wang Z, Xiong Y, Schorr C, et al. Impact of sepsis bundle strategy on outcomes of patients suffering from severe sepsis and septic shock in china. J Emerg Med 2013;44:735-41.

16. Zadroga R, Williams DN, Gottschall R, et al. Comparison of 2 blood culture media shows significant differences in bacterial recovery for patients on antimicrobial therapy. Clin Infect Dis 2013;56:790-7.

Cite this article as: Wang L, Ma X, He H, Su L, Guo Y, Shan G, Wang Y, Zhou X, Liu D, Long Y; China National Critical Care Quality Control Center Group. Compliance with the Surviving Sepsis Campaign guideline 1-hour bundle for septic shock in China in 2018. Ann Transl Med 2021;9(4):278. doi: 10.21037/atm-20-5429
17. Cardoso T, Carneiro AH, Ribeiro O, et al. Reducing mortality in severe sepsis with the implementation of a core 6-hour bundle: results from the Portuguese community-acquired sepsis study (SACiUCI study). Crit Care 2010;14:R83.

18. Kumar A. Systematic Bias in Meta-Analyses of Time to Antimicrobial in Sepsis Studies. Crit Care Med 2016;44:e234-5.

19. Levy MM, Rhodes A, Phillips GS, et al. Surviving Sepsis Campaign: association between performance metrics and outcomes in a 7.5-year study. Crit Care Med 2015;43:3-12.

20. Martin C, Viviand X, Leone M, et al. Effect of norepinephrine on the outcome of septic shock. Crit Care Med 2000;28:2758-65. 